\title{
Intra-operator Reliability of a 2D Image Analysing Method for Tooth Dimension Measurements
}

\author{
Kerekes-Máthé Bernadette1, Mártha Krisztina², Székely Melinda1 \\ ${ }^{1}$ Department of Morphology of Teeth and Dental Arches, Faculty of Dentistry, University of Medicine and Pharmacy, Tîrgu Mures, Romania \\ 2 Department of Paediatric Dentistry and Orthodontics, Faculty of Dentistry, University of Medicine and Pharmacy, Tîrgu Mureș, Romania
}

\begin{abstract}
Objective: The aim of this study was to evaluate the intra-operator reliability of a 2D image analysis method for tooth dimension measurements. Methods: Occlusal and vestibular surfaces of teeth from ten study models were captured with a digital camera. Images were transferred on a computer and different tooth dimensions (mesio-distal, occluso-gingival, vestibulo-oral and perimeter) were measured using the Image Pro Insight software. Measurements were repeated after two weeks. Fleiss' coefficients of reliability were calculated.

Results: A high degree of reliability has been established for every type of measurement: all coefficient values of reliability being not less than 0.95 .

Conclusions: The 2D image analyzing system is an adequate alternative for measuring tooth dimensions. All measurements were in the excellent category of reliability. The method allows precise and repeatable measurements, generating an image database as well, available for future studies.
\end{abstract}

Keywords: image analysing, tooth dimensions, reliability

Received: 22 May 2014 / Accepted: 11 June 2014

\section{Introduction}

Each measurement-based study must consider measurement errors. The technical error of measurement is the variability of different sizes in case of measuring the same specimen more than once. An experiment has a high degree of reliability if - given equivalent conditions - it is leading repeatedly to the same or similar results. Reliability refers to the closeness of the individual results from different sets of results, obtained by applying the method repeatedly on a homogeneous sample. There are two important factors that determine the reliability of the method: precision and accuracy. The precision of the measurement is the correlation between the values measured repeatedly on the same object, under the specified conditions. The accuracy of the method shows the degree of the closeness of the results to the true value. This refers to the reproducibility and repeatability of the measurements [1].

Factors which influence the reliability of tooth dimension measurements are the following: the operator; the procedure of taking the impression and making the stone cast; the condition of teeth and gingiva; measurement technique and tools. Repeated measurements of the same variable can not lead to the same results, due to the variability occured during the measurement process $[2,3]$.

The aim of this study was to evaluate the intra-operator reliability of a $2 \mathrm{D}$ image analysis method for tooth dimension measurements in order to use this method for further research at the University of Medicine and Pharmacy of Tîrgu Mureș.

\section{Methods}

Approval of the Research Ethics Committee of the University of Medicine and Pharmacy of Tîrgu-Mureş was obtained.

Upper and lower alginate (Ypeen Premium, SpofaDental) impressions were taken from healthy subjects, aged between 14-22 years. Dental stone models were obtained from these impressions (type 4 dental stone, FujiRock, GC). Ten study models have been selected for measurements. Inclusion criteria were: fully erupted permanent teeth and teeth without fillings or lesions.

Each tooth has been captured individually from vestibular and occlusal view with a digital camera (Nikon D3100, Nikon Corporation, Japan), using a $90 \mathrm{~mm}$ Tamron SP AF-S $90 \mathrm{~mm} \mathrm{f/2.8} \mathrm{macro} \mathrm{lens} \mathrm{having} \mathrm{a} \mathrm{1:1} \mathrm{magnification}$ ratio (Tamron). The camera lenses were focused parallel to the captured surface. An additional $55 \mathrm{~mm}$ circular polarizing filter (Hoya) was used to eliminate reflections and we used a cable shutter (Nikon MC-DC2). The camera was

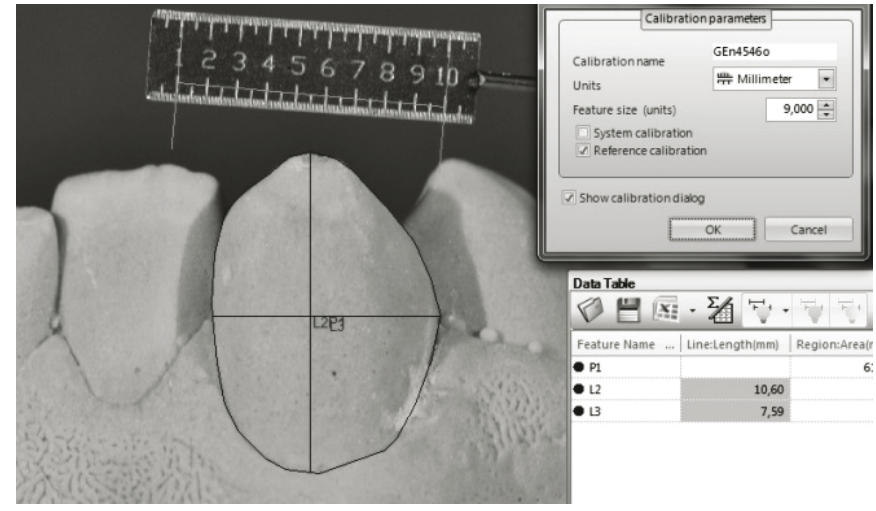

Fig. 1. Image calibration in the Image Pro Insight software 
Table I. Mean differences of the measured dimensions and the confidence levels

\begin{tabular}{lccc}
\hline \multirow{2}{*}{ Dimension } & Mean difference & \multicolumn{2}{c}{ Confidence levels } \\
\cline { 3 - 4 } & & Lower level & Upper level \\
\hline Vestibular view & & & \\
MD & 0.07 & 0.07 & 0.21 \\
OG & 0.07 & 0.09 & 0.23 \\
Perimeter & 0.13 & 0.07 & 0.33 \\
Occlusal view & & & \\
VO & 0.06 & 0.06 & 0.18 \\
Perimeter & 0.13 & 0.13 & 0.39
\end{tabular}

mounted horizontally above the model, on an adjustable stand (Kaiser 5360, Kaiser Fototechnik, Germany) with two halogen bulbs (190 W Tungsram, Hungary). Images were transferred with the ViewNX2 (Nikon Corporation, Japan) software and measurements were performed using Image Pro Insight software (Media Cybernetics, USA). A scale of ten millimeter was always positioned for subsequent image calibration (Figure 1).

After image calibration the following measurements were taken:

1. From vestibular view:

- mesio-distal (MD) dimension: the greatest distance between the mesial and distal surfaces of the dental crown;

- occluso-gingival (OG) dimension: the distance between the occlusal surface and gingiva, perpendicular to the MD line;

- tooth crown perimeter.

2. From occlusal view:

- vestibulo-oral (VO) dimension: the greatest distance between vestibular and oral surfaces;

- tooth crown perimeter.

At a two week distance all images were captured again and the same measurements were repeated, by the same operator.

Data obtained from measurements were processed using the SPSS statistical software Version 22 (IBM Corp.) and Fleiss' correlation coefficients were calculated.

\section{Results}

Images obtained for measurements had a 300 dpi resolution and a $4608 \times 3072$ pixels in size.

On the 10 study models a total number of 177 teeth were captured and measured twice. Mean difference was calculated between the two sets of measurements, separately for each dimension. The smallest differences between the pair measurements were found for the MD, OG and VO dimensions, with a value not more than $0.2 \mathrm{~mm}$ for each. (Table I).

In order to assess intra-operator repeatability, Fleiss' coefficients of reliability were calculated. Values higher than 0.6 represent a substantial reliability, while values higher than 0.8 are considered having an excellent reliability. A high
Table II. Fleiss' coefficients of reliability calculated separately for each tooth

\begin{tabular}{lccccc}
\hline Tooth & MD & OG & VO & PerimO & PerimV \\
\hline 1.1 & 0.997 & 0.998 & 0.99 & 0.996 & 0.998 \\
1.2 & 0.988 & 0.996 & 0.986 & 0.993 & 0.997 \\
1.3 & 0.967 & 0.995 & 0.996 & 0.996 & 0.998 \\
1.4 & 0.981 & 0.996 & 0.996 & 0.998 & 0.999 \\
1.5 & 0.994 & 0.998 & 0.995 & 0.999 & 0.999 \\
1.6 & 0.982 & 0.994 & 0.989 & 0.999 & 0.997 \\
2.1 & 0.99 & 0.994 & 0.995 & 0.998 & 0.999 \\
2.2 & 0.988 & 0.997 & 0.989 & 0.998 & 0.998 \\
2.3 & 0.976 & 0.997 & 0.996 & 0.997 & 0.999 \\
2.4 & 0.963 & 0.997 & 0.991 & 0.996 & 0.999 \\
2.5 & 0.985 & 0.997 & 0.992 & 0.997 & 0.998 \\
2.6 & 0.984 & 0.995 & 0.986 & 0.997 & 0.998 \\
3.1 & 0.988 & 0.992 & 0.976 & 0.993 & 0.997 \\
3.2 & 0.983 & 0.998 & 0.988 & 0.992 & 0.993 \\
3.3 & 0.986 & 0.992 & 0.994 & 0.995 & 0.997 \\
3.4 & 0.979 & 0.985 & 0.984 & 0.996 & 0.997 \\
3.5 & 0.997 & 0.999 & 0.995 & 0.997 & 0.999 \\
3.6 & 0.988 & 0.99 & 0.986 & 0.992 & 0.999 \\
4.1 & 0.974 & 0.996 & 0.953 & 0.971 & 0.998 \\
4.2 & 0.986 & 0.996 & 0.997 & 0.993 & 0.995 \\
4.3 & 0.979 & 0.995 & 0.996 & 0.996 & 0.998 \\
4.4 & 0.984 & 0.995 & 0.993 & 0.997 & 0.997 \\
4.5 & 0.989 & 0.995 & 0.995 & 0.998 & 0.999 \\
4.6 & 0.991 & 0.99 & 0.978 & 0.998 & 0.998 \\
\hline & & & & &
\end{tabular}

degree of reliability has been established for every type of measurement, all values being higher than 0.95 (Table II).

\section{Discussion}

Any given measurement method can result in a technical error of measurement. For this reason, measurement-based studies are preceded by reliability studies, which will assess the repeatability of the measurements [1]. These studies provide information on intra- and interoperator measurement errors. In the case of a 2D image analysis method, the potential source of errors could be represented by the poor quality of the digital image, the used calibration scale, the angle-of-view of the image, as well as the identification of the different measurement reference points $[4,5]$.

Reduction of the technical error of measurement is possible by improving either the measuring tool or the measurement technique.

The traditional method for the measurement of tooth dimensions is accomplished by the use of calipers. Dental measurements are important in different fields of dental medicine, as well as in the etiological study of dental anomalies. Much more detailed and complex measurements would be required in order to achieve an ever increasing understanding of the etiology of dental anomalies [6].

The bidimensional image analysis system has many advantages over the currently employed method: allows the measurement of some supplementary parameters, such as area, perimeter and subdivisions of the teeth from any given angle-of-view, allows extensive data and image storage and later measurements under the same conditions. This method has been developed and validated by Brook et al. $[7,8]$. 
The digital camera provided high resolution images. The time for capturing a tooth surface took about three minutes and the time to perform measurements on an image, i.e. one surface of a single tooth, was about one minute. It is possible to include two teeth on the same image, on the condition that the entire surface of both teeth is visible and these surfaces are in the same plane.

Data from published scientific literature showed high or excellent correlation coefficients for the $2 \mathrm{D}$ image analysis method of investigation $[6,9,10]$. Our findings regarding the intra-operator repeatability of this method were similar: every type of measurement had an excellent coefficient of reliability.

For studies involving more than one operator, it is necessary to assess also the inter-operator reliability of the method $[6,10]$. For this study this assessment has not been carried out, since only one operator was involved. For a larger scale, future employment of this method, an interoperator reliability study will be mandatory.

\section{Conclusions}

The $2 \mathrm{D}$ image analyzing system is an adequate alternative for measuring tooth dimensions. All measurements, from occlusal and vestibular view, were in the excellent category of reliability. This method allows precise and repeatable measurements, generating an image database available for future studies as well.

\section{Acknowledgments}

This study was supported by the Internal Research Grant no. 8/30.01.2013 of the University of Medicine and Pharmacy of Tîrgu Mureș, Romania.

\section{References}

1. Harris E, Smith RN. Accounting for measurement error: a critical but often overlooked process, Archives of oral biology. 2009;54S:107-117.

2. Bland JM, Altman DG. Statistical notes: measurement error. BMJ. 1996;313:744.1.

3. WHO Multicentre Growth Reference Study Group. Reliability of anthropometric measurements in the WHO Multicentre Growth Reference Study. Acta Paediatrica. 2006;450:38-46.

4. Smith R, Zaitoun $\mathrm{H}$, Coxon $\mathrm{T}$, et al. Defining new dental phenotypes using 3-D image analysis to enhance discrimination and insights into biological processes, Archives of Oral Biology. 2009;54(S1):S118-S125.

5. Robinson DL, Blackwell PG, Stillman EC, Brook AH. Planar Procrustes analysis of toothshape. Archives of Oral Biology. 2001;46(3):191-199.

6. Brook $A H$, Griffin $R C$, Smith $R N$, et al. Tooth size patterns in patients with hypodontia and supernumerary teeth, Archives of Oral Biology. 2009;54(1):S63-S70.

7. Brook A, Smith R, Elcock C, et al. The measurement of tooth morphology: development and validation of a new image analysis system. In: Mayhall J, Heikkinen T, editors. Proceedings of the $11^{\text {th }}$ international symposium on dental morphology. Oulu: Oulu University Press; 1998:380-387.

8. Al-Sharood M. Measurement of tooth size and shape in subjects with hypodontia and a control group using a new image analysis technique, Ph.D. Thesis, The Univerity of Sheffield, 2000.

9. McKeown HF, Robinson DL, Elcock C, Al-Sharood M, Brook AH. Tooth dimensions in hypodontia patients, their unaffected relatives and a control group measured by a new image analysis system. European Journal of Orthodontics. 2002;24:131-141.

10. Brook AH, Elcock C, Aggarwal M, et al. Tooth dimensions in hypodontia with a known PAX9 mutation, Archives of Oral Biology. 2009;54(1): S57-S62. 